\title{
Wave modes from the magnetorotational instability in accretion discs
}

\author{
Giuseppe Di Bernardo ${ }^{1,2}$ and Ulf Torkelsson ${ }^{1}$ \\ ${ }^{1}$ Department of Physics, University of Gothenburg, \\ SE 41296 Gothenburg, Sweden \\ email: giuseppe.dibernardo@physics.gu.se, torkel@chalmers.se \\ ${ }^{2}$ Department of Astronomy and Theoretical Astrophysics Center, University of California \\ Berkeley, \\ Berkeley, CA 94720 \\ email: giuseppe.dibernardo@berkeley.edu
}

\begin{abstract}
The magnetorotational instability (MRI) is widely believed to be the source of turbulence in accretion discs. This turbulence is responsible for the anomalous angular momentum transport in accretion discs. The turbulence will affect other aspects of the dynamics of the disc as well, and we will concentrate on two such issues: a) what kind of oscillations can be excited by the turbulence itself, and b) how the turbulence is interacting with modes that have been excited by some other agent. This is of interest in understanding the quasi-periodic oscillations (QPOs) that have been observed in the X-ray light curves of accreting neutron star and black hole binaries. We carry out local three dimensional (3D) magnetohydrodynamic simulations of a keplerian differentially rotating accretion disc, using a shearing box configuration taking in account the effects of the vertical stratification.
\end{abstract}

Keywords. Accretion discs, instability, MHD, turbulence, wave, methods: numerical.

Kilohertz quasi-periodic oscillations (KHz QPOs) are often observed in pairs, usually called lower and upper $\mathrm{kHz} \mathrm{QPO}$, with frequencies ranging from a few hundred $\mathrm{Hz}$ to more than 1 kilohertz. In the past 16 years, several models have been proposed to explain these oscillations, with an emphasis on trying to explain the frequencies of the QPOs. Almost all models associate the frequencies of these oscillations with characteristic frequencies in a geometrically thin accretion disc. An equally important issue is to understand how the oscillatory modes are excited.

Balbus \& Hawley (1991) showed more than twenty years ago that differentially rotating accretion discs are linearly unstable in the presence of a weak magnetic field. The hydromagnetic turbulence which derives from the magneto-rotational instability (MRI, also known as Balbus-Hawley instability) is generally considered as the most promising source for angular momentum transport in accretion flows. During these years there has been significant progress in developing numerical simulations that demonstrate the viability of this concept, and how the turbulence leads to a self-sustained dynamo (Brandenburg et al. 1995). At the same time there have been only a few works (Brandenburg 2005) that have looked at the excitation of oscillatory modes in the turbulence. In this paper we return to this problem, taking into account the effect of the vertical stratification of the disc. Our simulations confirm that the turbulence does indeed excite radial epicyclic oscillations and axisymmetric acoustic modes (Arras et al. 2006).

All of the work described here is based on numerical setups implemented in the PENCIL CODE $\dagger$ which uses a sixth order centered finite-difference in space, and a third-order

$\dagger$ http://pencil-code.googlecode.com 

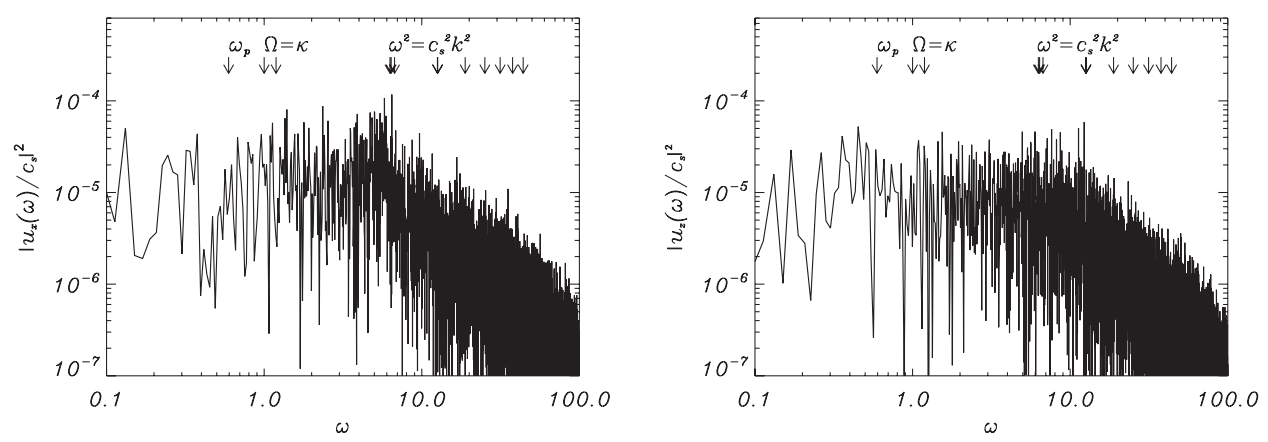

Figure 1. Power spectra for radial (left panel) and vertical velocity fluctuations (right panel), recorded at the midplane of the box $(z=0)$. Distinct peaks in the power spectrum are shown as arrows. These peaks can be identified with certain classes of hydrodynamic modes as discussed in the text. The frequency of standing sound waves (p-modes) is marked by an arrow below the epicyclic frequency. From left to right, axisymmetric acoustic modes lie above the epicyclic frequency.

Runge-Kutta scheme in time. In a local patch of a Keplerian accretion disc, we solve the governing equations of MHD for an isothermal equation of state, and adopting the shearing formalism. Typically the computational domain has dimensions of $1 \mathrm{H} \times 4 \mathrm{H} \times 5.28 \mathrm{H}$ in the radial, azimuthal and vertical dimensions, where $H$ is the isothermal Gaussian scale height. Finally, an initial weak toroidal magnetic field, $\boldsymbol{A}=A_{0} \cos \left(k_{A} x\right) \cos \left(k_{A} y\right)$ $\cos \left(k_{A} z\right) \mathbf{e}_{\mathbf{y}}$, will thread the box. A linear perturbation of a time-indipendent keplerian shear flow predicts that axisymmetric inertial-acoustic modes behave like $\omega^{2}=\kappa^{2} k_{z}^{2} / k^{2}$ (inertial modes), at the lowest wavenumbers in our simulations, while above the epicyclic frequency their dispersion relationship is that of acoustic modes, $\omega^{2}=c_{s}^{2} k^{2}$ (Arras et al. 2006). In Fig.1 we show the power spectra for radial and vertical velocity fluctuations computed in our simulations, while the arrows represent the position of the waves expected from the linear theory mentioned above. As can be noticed by Fig.1, axisymmetric acoustic normal modes and a global axisymmetric epicyclic oscillation at the orbital frequency are clearly reproduced in our numerical data. However, hydrodynamic inertial waves modes that have been proposed as the origin of some observed QPOs in X-ray binaries are not clearly detected.

We conclude, emphasizing that including the vertical gravity profile and a proper set of boundary conditions, we correctly reproduced the nonlinear behaviour necessary for the angular momentum transport. Our simulations aimed to investigate oscillatory modes which might occur in accretion discs. As a natural follow-up we plan to include a proper cooling function in order to represent astrophysical disc systems more accurately.

\section{References}

Arras, P., Blaes, O., \& Turner, N. J. 2006, ApJ, 645, 65

Balbus, S. A \& Hawley, J. F. 1991, ApJ, 376, 214

Brandenburg, A., Nordulund, A., Stein, R. F., \& Torkelsson, U. 1995, ApJ, 446, 741

Hawley, J. F., Gammie, C. F., \& Balbus, S. A. 1995, ApJ, 440, 742 